\title{
OPEN Enhanced genome editing efficiency of CRISPR PLUS: Cas9 chimeric fusion proteins
}

\author{
Jongjin Park ${ }^{1,2,6}$, Jiyoung Yoon ${ }^{1,6}$, Daekee Kwon ${ }^{3}$, Mi-Jung Han $^{3}$, Sunmee Choi ${ }^{1}$, Slki Park ${ }^{1}$, \\ Junghyuk Lee ${ }^{1}$, Kiwook Lee ${ }^{1}$, Jaehwan Lee ${ }^{1}$, Seunghee Lee ${ }^{3}$, Kyung-Sun Kang ${ }^{3,4 \bowtie}$ \& \\ Sunghwa Choe $\mathrm{C}^{1,2,5 \bowtie}$
}

Efforts to improve CRISPR-Cas9 genome editing systems for lower off-target effects are mostly at the cost of its robust on-target efficiency. To enhance both accuracy and efficiency, we created chimeric SpyCas9 proteins fused with the 5'-to-3' exonuclease Recombination J (RecJ) or with GFP and demonstrated that transfection of the pre-assembled ribonucleoprotein of the two chimeric proteins into human or plant cells resulted in greater targeted mutagenesis efficiency up to $600 \%$ without noticeable increase in off-target effects. Improved activity of the two fusion proteins should enable editing of the previously hard-to-edit genes and thus readily obtaining the cells with designer traits.

Clustered regularly interspaced short palindromic repeats (CRISPR) and CRISPR-associated 9 (Cas9) constitute an adaptive immune system found in bacteria, archaebacteria, and bacteriophages ${ }^{1-3}$, but thanks to its programmability and ease of use, CRISPR-Cas9 has been repurposed for genome editing in various applications, including research, therapeutics, microbiome manipulation, and agriculture ${ }^{4-8}$.

Within the CRISPR-Cas9 system, the functional RNA-Cas9 complex consists of a Cas9 effector protein that acts as a DNA cleaving enzyme and two single strand guide RNAs, namely, a trans-acting CRISPR RNA (tracrRNA) and a CRISPR RNA (crRNA), which guide the complex to the target sequence. The RNA-Cas9 ribonucleoprotein (RNP) complex localizes to its target sequence in two steps; it first locates and binds to the protospacer adjacent motif (PAM) (5'-NGG-3' for Cas9) of the nontarget strand and then unwinds the target DNA to complementarily bind between crRNA and the target strand of the protospacer sequence in the genome. In eukaryotic cells, the dsDNA breaks (DSB) created in the target DNA by Cas9 RNP are repaired by either homology-directed repair (HDR) or nonhomologous end joining (NHEJ) ${ }^{9}$. Error-prone NHEJ process can produce insertion and/ or deletion (indel) mutations of variable length at the target site, whereas HDR-mediated repair can replace the DNA sequence with the help of a single-stranded or double-stranded DNA donor template.

The Cas9 protein from Streptococcus pyogenes (SpyCas9) has been extensively characterized and developed for various applications since initial demonstration in $2012^{1,10}$. Various steps in the mode of action of the SpyCas 9 RNP have been targeted for improvement, including protein engineering of the domains interacting with PAM, nontarget strands, and crRNA, which enabled specificity to be improved ${ }^{11}$; furthermore, chemical modifications to the guide RNA for stability have improved SpyCas9 function ${ }^{12}$. However, efforts to enhance efficiency are relatively unattended. Enzyme kinetics is affected by both how fast the enzyme (E) binds to the substrate (S) to form ES complex that leads to an enzyme-product (EP) complex, and also by how fast the enzyme dissociates from the EP complex and hits another substrate ${ }^{11}$. Different from conventional enzymes, the energetically stable complex between the SpyCas9 RNP and protospacer DNA makes the ternary RNA-protein-DNA structure difficult to dissociate, and this property makes Cas9 a single-turnover enzyme that does not follow the conventional Michaelis and Menten kinetics ${ }^{13}$.

In an attempt to enhance the enzymatic activity of SpyCas9, various proteins including endonucleases, exonucleases, ligase inhibitors, deaminases, and an RNA reverse transcriptase have been translationally fused to the SpyCas9 protein. When an E. coli 3' to $5^{\prime}$ Exonuclease I ( $\mathrm{sbcB}$ ) was fused to SpyCas9, the chimeric protein

\footnotetext{
${ }^{1}$ G+FLAS Life Sciences, CRISPR PLUS Lab, 38 Nakseong-daero, Gwanak-Gu, Seoul 08790, Korea. ${ }^{2}$ Naturegenic Inc, 1281 Win Hentschel Boulevard, Kurz Purdue Technology Center Suite 1573, West Lafayette, IN 47906, USA. ${ }^{3}$ Stem Cells and Regenerative Bioengineering Institute in Kangstem Biotech, Gwangmyeong SK TechnoPark, Gwangmyeong-si 14322, Gyeonggi-do, Korea. ${ }^{4}$ Adult Stem Cell Research Center, College of Veterinary Medicine, Seoul National University, Seoul 08826, Korea. ${ }^{5}$ School of Biological Sciences, College of Natural Sciences, Seoul National University, Gwanak-gu, Seoul 08826, Korea. ${ }^{6}$ These authors contributed equally: Jongjin Park and Jiyoung Yoon. ${ }^{\square}$ email: kangpub@snu.ac.kr; shchoe@snu.ac.kr
} 
generated the longer deletions of DNA in Zebrafish relative to SpyCas9 alone ${ }^{14}$. In addition, a SpyCas9 chimeric fusion protein with the three prime repair exonuclease 2 (TREX2), a human $3^{\prime}$ to $5^{\prime}$ exonuclease involved in DNA repair, replication, and recombination, was also reported to increase mutagenic efficiency ${ }^{15,16}$. TEXT (Tethering EXonuclease T5 with FnCas12a) - a fusion strategy significantly increased the knockout efficiency of FnCas12a at multiple genomic loci in different human cell lines ${ }^{17}$. In case of the knock-in activity, when the human CtIP endonuclease was fused to SpyCas9, the chimeric protein resulted in more than two-fold increase in transgene integration efficiency relative to SpyCas9 backbone protein alone ${ }^{18}$. A dominant-negative mutant of 53BP1, DN1S, was fused to Cas9, and the Cas9-DN1S fusion proteins significantly blocked NHEJ events specifically at Cas 9 cut sites and improved HDR frequency ${ }^{19}$. Rad52-Cas9 fusion strategies yielded approximately threefold increase in HDR during the surrogate reporter assays in human HEK293T cells ${ }^{20}$. Furthermore, the chimeric fusion of adenine or cytidine deaminases to nuclease-inactivated version of SpyCas 9 resulted in base editing without DSB, concomitantly reducing unintended off-target effects ${ }^{21,22}$. Lastly, an engineered reverse transcriptase fused to a catalytically-impaired SpyCas9 and a prime editing-guide RNA (pegRNA) led to specific change of the base using the pegRNA as a repair emplate ${ }^{23}$.

Because the chimeric fusion proteins with the SpyCas9 were shown to widen the spectrum of capability of the site-specific nuclease SpyCas9. We attempted to test the accessary proteins that may weaken the binding energy of the chimeric proteins to the substrate DNA and thus increase a turnover rate to eventually enhance the genome editing efficiency. To this end, we first created fusion proteins in which the carboxy-terminus of SpyCas9 was linked with a DNA-modifying protein-either a 5' to 3' DNA exonuclease (RecE, RecJ, T5, or lambda) ${ }^{24}$, mung bean nuclease, or terminal deoxynucleotidyl transferase (TdT). T5 and RecJ exonucleases were reported to enhance FnCas12a, T5-FnCas12a increased the knockout efficiency of FnCas12a at multiple genomic loci in different human cell lines, while RecJ-FnCas12a decreased the knockout efficiency of FnCas $12 \mathrm{a}^{17}$.

The green fluorescent protein (GFP) was also tested as a control. Of the seven tested, SpyCas9 fusion with RecJ (SpyCas9-RecJ, C9R) and surprisingly with GFP (SpyCas9-GFP, C9G) resulted in a marked increase in both mutagenesis and knock-in efficiency, while the off-target effects are not significantly increased relative to the SpyCas9 (C9) structure alone. We refer the two functionally enhanced fusion proteins of C9R and C9G to as CRISPR PLUS, and explore how efficiently they edit the DNA at different conditions such as in test tube, cultured cell line, primary human cells, and plant protoplasts.

\section{Results and discussion}

Increased genome-editing efficiency of Cas9 chimeric fusion with RecJ or GFP. To prepare Cas9 chimeric fusion proteins, we translationally fused the carboxy terminus of SpyCas 9 to a modifier protein: $5^{\prime}$-to3' DNA exonucleases (including RecE, Rec), T5, and lambda), mung bean nuclease, TdT, or GFP (Fig. 1A) (S1 Text). The SpyCas 9 chimeric fusion proteins, were expressed in bacterial cells and purified. The SDS-PAGE pattern shows that all chimeras were isolated as single proteins (S1 Fig), indicating successful fusion of the accessory proteins and purity to perform functional analysis. The SpyCas9-T5 protein was barely detectable $(<0.2 \mathrm{mg} / \mathrm{L})$ due to its low expression level.

To investigate the activity of the seven fusion proteins, we performed an in vitro DNA cleavage assay with the therapeutically relevant loci examined by other colleagues for cross comparison ${ }^{25}$. The $1.5 \mathrm{~kb}$ PCR-amplified $C-C$ chemokine receptor type 5 (CCR5) DNA was cut in vitro by C9 and the CRISPR PLUS proteins, 750 bp DNA fragments were produced (Fig. 1B). A 0.25 pmol of each SpyCas9 fusion protein and a 0.3 pmol of sgRNA CCR5 were mixed and incubated at room temperature for $15 \mathrm{~min}$. A total of $250 \mathrm{ng}$ of DNA was digested with preassembled RNP at $37^{\circ} \mathrm{C}$ for $10 \mathrm{~min}$. The enzymatic products were then quantified from the agarose gel using Image J and the corresponding enzymatic activities were calculated. C9 exhibited $14.1 \%$ cleavage activity, whereas C9R and C9G showed $40.6 \%$ and $26.3 \%$ activity, respectively (Fig. 1C). By contrast, fusion with RecE resulted in difficulty purifying the proteins possibly due to instability of C9-RecE chimeric fusion structure, to $1.3 \%$, and fusion with lambda, mung bean nuclease, and hTdT did not significantly affect the C9 activity. Together, these results demonstrate that the fused fragments affect the overall enzymatic activities of the Cas9. Henceforth, we further analyzed only C9R and C9G due to their desired effects on activity.

Improved indel efficiency of CRISPR PLUS in the cells. To check whether C9R induces DNA insertion and deletion (Indel) mutations more rapidly than does C9, we first confirmed that the RecJ moiety of C9R is functional in vitro and fluorescence activity of GFP domain of C9G (S2 Fig.). We then introduced C9, C9R, and C9G ribonucleoproteins (RNPs) targeting the CCR5 gene into HEK293T cells and stopped the incubation at 8, 16 , or $24 \mathrm{~h}$ after transfection; cells were then harvested and subjected to a T7 endonuclease 1 assay, the DNA was resolved on a $2 \%$ gel, and the results of the three replicates were digitized using ImageJ. C9R and C9G reached $20 \%$ efficiency as early as $8 \mathrm{~h}$, a level that was not achieved until at least $16 \mathrm{~h}$ for the C9 control (Fig. 1D). Relative to C9, CRISPR PLUS displayed approximately twofold increase in efficiency at $16 \mathrm{~h}$.

To analyze the enzymatic activity in relation to different gene targets, we transfected the preassembled RNPs of C9, C9R, and C9G targeting CCR5 into HEK293T cells and incubated them for $24 \mathrm{~h}$; the indel efficiency was 39\%, 76\%, and 87\%, respectively (Fig. 1E). Meanwhile, C9, C9R, and C9G displayed efficiencies of 22\%, 59\%, and $43 \%$, respectively, for hypoxanthine phosphoribosyltransferase 1 (HPRT1) and 7\%, 36\%, and 42\% for Empty Spiracles Homeobox 1 (EMX1; Fig. 1E). Overall, C9R displayed approximately two- to fivefold increased efficiency; C9G also exhibited two- to sixfold increased activity relative to C9.

Because the RecJ moiety of C9R as a $5^{\prime}$ to $3^{\prime}$ ssDNA exonuclease resects DNA from $5^{\prime}$ to $3^{\prime}$ direction, the deletion created by C9R at DSB could be longer that that by C9. To determine whether the increased indel efficiencies of the CRISPR PLUS proteins are associated with deletion size, we first analyzed the correlation between deletion size and the number of NGS-based deep sequencing reads (>40,000 per locus) at the CCR5 target 

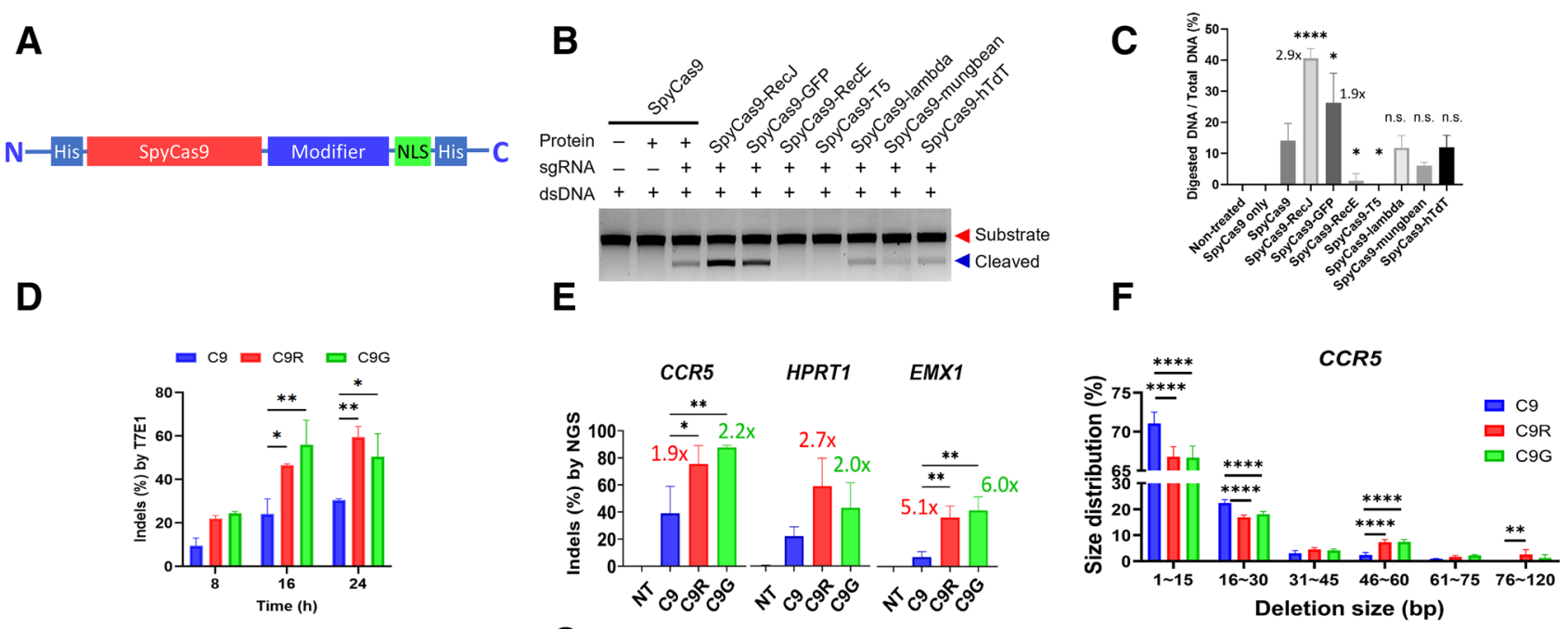

G

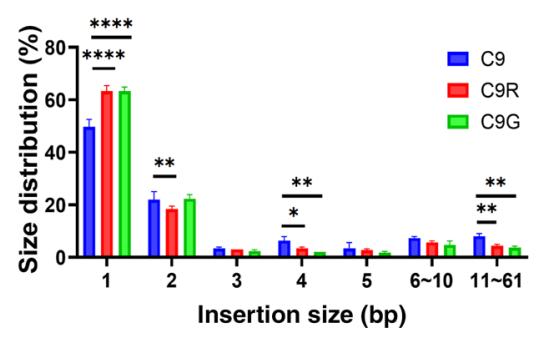

Figure 1. Marked increase in indel efficiency of the Cas9-RecJ and Cas9-GFP chimeric fusion proteins. (A) Schematic of a translational fusion of a modifier protein to SpyCas9. His tag (6X His) for protein purification and nuclear localization signal (NLS) were placed. (B) Comparison of the DNA cleavage activity of SpyCas9 and its chimeric proteins, C-terminal chimeric SpyCas9 translational fusion proteins with the nucleases RecJ, RecE, T5, lambda, mung bean exonuclease, and human terminal deoxynucleotidyl transferase (TdT), and with green fluorescent protein (GFP). The modifier proteins are described in Supplementary Table 1. The CCR5 DNA was digested with the purified enzymes and resolved on an agarose gel. Successful digestion cuts the $1.5 \mathrm{~kb}$ substrate DNA into two $0.75 \mathrm{~kb}$ DNAs. (C) DNA band intensities in the gel image. Statistical comparison was made between C9 and each of the C9R or C9G proteins. Significance was indicated on top of each bar. One-way ANOVA using Tukey's multiple-comparison test. The fold change relative to C9 is indicated above each bar. (D) DNA insertion and deletion (Indel) efficiency of C9, C9R, and C9G for the CCR5 gene in HEK293T cells at 8, 16, and $24 \mathrm{~h}$ after RNP transfection as measured by a T7E1 assay. Averages from the three replicate experiments are plotted. Two-way ANOVA using Tukey's multiple-comparison test. (E) Indel efficiency of C9, C9R, and C9G for the CCR5, HPRT1, and EMX1 genes (left to right) in HEK293T cells. At $24 \mathrm{~h}$ (CCR5 and HPRT1) and $8 \mathrm{~h}$ $(E M X 1)$ post-transfection, cells were harvested and subjected to targeted deep sequencing. The experiments were performed in triplicate. NT group represent the NGS data from non-treated control. One-way ANOVA using Tukey's multiple-comparison test was performed using C9 data as control. (F) Distribution of the DNA deletion sizes after treatment with C9, C9R, and C9G. The number of deletion events around the protospacer site in the CCR5 gene was counted according to the $15 \mathrm{bp}$ deletion intervals from targeted deep sequencing data $(n=3)$ data. (G) Distribution of DNA insertion sizes after treatment with C9, C9R, and C9G in the CCR5 gene. The number of insertion events was counted according to the $1 \mathrm{bp}$ insertion intervals from targeted deep sequencing data $(\mathrm{n}=3)$ data. 6-10 and 11-61 bp insertion intervals were also shown to include events with smaller numbers. ${ }^{* * *} P<0.0001,{ }^{* *} P<0.001,{ }^{* \star} P<0.01,{ }^{\star} P<0.05$, and n.s. indicates not significant.

locus. Compared to C9, CRISPR PLUS exhibited significantly reduced percentages of small deletions of 1-15 and 16-30 bp (Fig. 1F), but significant increases in longer DNA deletions of 46-60 and 76-120 bp. Conversely, insertions tended to be shorter than those using C9 (Fig. 1G).

Next, we evaluated the off-target effects of CRISPR PLUS. Five potential off-target sequences of the CCR5, HPRT1, and EMX1 genes were identified by Cas-OFFinder ${ }^{7}$ (S3 Fig). For sequences with $>50,000$ NGS reads, the error rates of CRISPR PLUS did not exceed that of conventional NGS sequencing error rate (0.01-0.1\%) (S3 Fig), suggesting that the high on-target editing efficiency of CRISPR PLUS is accompanied by low off-target effects.

Taken together, we demonstrated that CRISPR PLUS consistently displayed greater efficiency in indel mutagenesis in three different genes of HEK293T cells, whereas the off-target effects are not increased as compared with C9 treatment. Now we turn to testing knock-in efficiency of the CRISPR PLUS proteins. 
A 106-mer ssODN

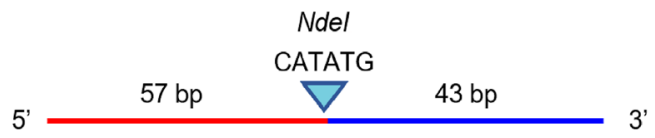

B
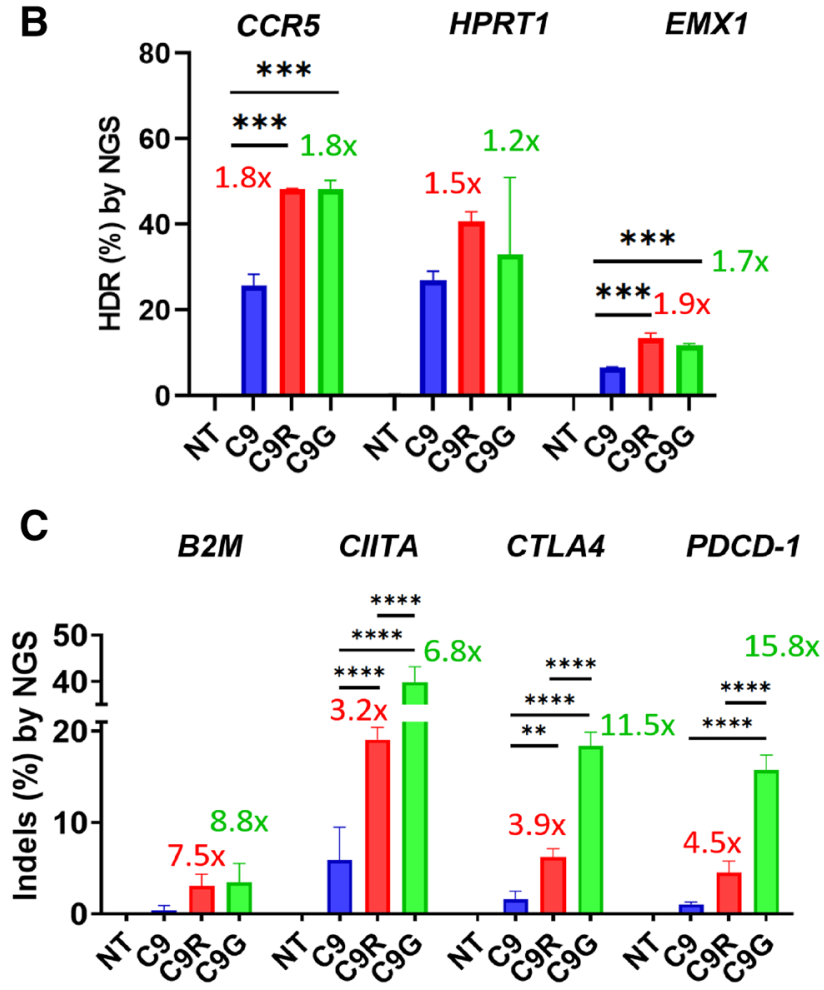

Figure 2. Increase in knock-in efficiency in HEK293T cells and enhanced multiplex indel efficiency of chimeric proteins in iPSCs. (A) Schematic of a 106-mer single-stranded oligonucleotide (ssODN). Artificially introduced NdeI restriction site (blue triangle) was flanked with homologous 5' arm (57-mer) and 3' arm (43-mer). (B) Comparison of homology-directed DNA repair (HDR) activity among C9, C9R, and C9G. HDR efficiency was evaluated by targeted deep sequencing of the CCR5, HPRT1, and EMX1 loci (left to right) after genome editing with the specific 106-mer template shown in panel a. ${ }^{* * * *} P<0.0001$ and ${ }^{* * *} P<0.001$, one-way ANOVA using Tukey's multiple-comparison test). For each gene, fold change was calculated relative to C9 efficiency. (C) Four different RNPs, each preassembled with a different sgRNA (targeting B2M, CIITA, CTLA4, or PDCD-1) were simultaneously transfected into induced pluripotent stem cells (iPSCs) and incubated for $24 \mathrm{~h}$, and the targeted deep-sequencing analysis was performed to determine the indel efficiencies $\left({ }^{* * * *} P<0.0001,{ }^{* *} P<0.001\right.$, and ${ }^{*} P<0.01$, two-way ANOVA using Tukey's multiple-comparison test).

Enhanced homology-directed DNA repair (HDR) of CRISPR PLUS. In addition to indel mutagenesis, knock-in replacement of the sequence is the major area of CRISPR-Cas9 applications. We therefore investigated whether CRISPR PLUS improves HDR activity for the three target genes in HEK293T cells. The cell cycle was first synchronized using nocodazole ${ }^{26}$ before transfection with both the preassembled RNP complex (protein:sgRNA = 30:60 pmol) and a 106-mer single strand oligodeoxynucleotide (ssODN) repair template (20 pmol) consisting of a 57-nt 5' homology arm, 5'-CATATG-3' NdeI restriction enzyme site, and 43-nt 3' homology arm (Fig. 2A). Each target site was amplified and analyzed using targeted amplicon sequencing, and the HDR efficiencies were evaluated by NdeI digestion of the amplified DNA (S4 Fig). C9 exhibited 20\%, 23\%, and 5\% efficiency for the CCR5, HPRT1, and EMX1 sites, respectively; these values were 36\%, $48 \%$, and $12 \%$ for C9R and 36\%, 39\%, and 11\% for C9G (S4 Fig). Therefore, relative to C9, CRISPR PLUS increased the knock-in replacement efficiency by $6-16 \%$. When the efficiency was re-examined using targeted deep sequencing analysis, the efficiency of C9 for the CCR5, HPRT1, and EMX1 sites was found to be $26 \%, 27 \%$, and $6 \%$, respectively; that of C9R was $48 \%, 41 \%$, and $13 \%$; and that of C9G was $48 \%, 33 \%$, and $12 \%$, confirming that CRISPR PLUS increased the knock-in efficiency by $6-22 \%$ (Fig. 2B). It is likely that increased knock-in efficiency might be associated with the increased editing activity of CRISPR PLUS enzymes.

Increased multiplex genome editing efficiency in induced pluripotent stem cells (iPSCs) and plant protoplasts. Finally, to determine whether the enhanced genome editing efficiency might be appli- 
cable to induced pluripotent stem cells (iPSCs), a cell type that is recalcitrant to editing, we performed multiplex genome editing of the beta 2 microglobulin (B2M), class II major histocompatibility complex transactivator (CIITA), cytotoxic T-lymphocyte-associated antigen 4 (CTLA4), and programmed cell death protein 1 (PDCD1). When it comes to regenerative medicine using universal iPSCs, deletion of B2M and CIITA in iPSCs suppresses expression of their human leucocyte antigen (HLA) Class I and Class II genes, respectively, such that the genome edited iPSCs can avoid host T cell-mediated clearance ${ }^{27}$. CTLA4 and PDCD1 are inhibitory receptors that take part in $\mathrm{T}$ cell exhaustion, therefore being the target of deletion when designing engineered $\mathrm{T}$ cells for cancer immunotherapy ${ }^{28}$. We first designed two sgRNAs for each of the four genes, tested in HEK293T cells, and selected the more efficient sgRNA from each pair, where $\operatorname{sgRNA}^{\mathrm{B} 2 \mathrm{M}}-2$, sgRNA ${ }^{\mathrm{CITA}_{-}}-1, \operatorname{sgRNA}^{\mathrm{PDCD} 1}-1$, and sgRNA ${ }^{\text {CTLA4 }}$-1 were chosen for the iPSC experiments (S5 Fig).

After in vitro assembly of each of the four sgRNAs with effector proteins, namely, C9, C9R, and C9G, three sets of four RNPs were transfected into HEK293T before moving to iPSCs. At three days post-electroporation, T7 endonuclease I assay (T7E1) was performed. Three-day treatment resulted in indel efficiency $38-81 \%$ regardless of C9, C9R, and C9G (S6 Fig). In iPSCs, compared with C9, CRISPR PLUS increased indel efficiency 7.5-fold and 8.8-fold for B2M (Fig. 2C), 3.2- and 6.8-fold for CIITA, 3.9- and 11.5-fold for CTLA4, and 4.5- and 15.8-fold for $P D C D-1$, respectively, suggesting possibility to obtain multiplex homozygous mutations in the target genes within shorter time than using C9.

Plant protoplasts are an example of difficult-to-edit cells partly because the nucleofection of the RNP complex damages the cells. Therefore, obtaining desired level of editing efficiency with a tolerable amount of RNP is a demanding technique. To determine whether C9R activity is reproducible in plants, C9 and C9R RNPs programmed to target $\alpha$-1,3-fucosyltransferase 1 were transfected into protoplasts of the model plant Nicotiana benthamiana by the DNA-free genome editing method (as described in previous studies ${ }^{29}$ ), and their editing efficiency were analyzed by NGS deep sequencing. Compared to C9, C9R had increased editing efficiency by 2.6-fold, (S7 Fig), suggesting that C9R activity is conserved regardless of the plant or animal system.

Taken together, we have shown that the two CRISPR PLUS proteins display markedly increased efficiency in both indel mutagenesis and knock-in efficiency at the various conditions tested such as in vitro DNA cleavage, in plant protoplasts, human cells, and in iPSC cells. However, currently, we do not have a complete explanation about the mechanisms of action of the enhanced genome editing efficiency observed from CRISPR PLUS especially in the C9G protein. In fact, it was not too much surprising that the C9R showed greater efficiency relative to C9 alone, because RecJ moiety could contribute to leaving permanent mutation footprint at the site of DSB. However, it was indeed unexpected results obtained from C9G experiments.

To rule out any possibility of experimental errors, we examined the identity of the C9G protein and performed the editing experiments repeatedly with separately prepared batches of C9G, but obtained basically the same results. First, we double checked if our C9G protein surely has GFP moiety by examining the photochemical spectra of both C9 and C9G. Specific light absorption and emission of C9G peaked around 400 and $500 \mathrm{~nm}$, respectively as expected, whereas $\mathrm{C} 9$ did not noticeably absorb or emit at these wavelengths (S2 Fig. B). Emission of green light from $\mathrm{C} 9 \mathrm{G}$ protein confirms that we used the right proteins. In addition, it can be postulated that the GFP moiety might contribute to stabilizing the C9G protein, and the relatively stable C9G acts longer time than $\mathrm{C} 9$ to result in overall increase in genome editing efficiency. However, our data shows that the activity was found enhanced at as early as $16 \mathrm{~h}$, and not significantly increased then after (Fig. 1D). On the other hand, it is also of note that the nucleotide deletion size of both C9R and C9G similarly decreased at smaller size (1-30 nt) but increased at larger section (46-60 nt) (Fig. 1F). Thus, the mode of action of C9G could be similar to C9R, but it needs further research to draw definitive conclusion about the enhanced genome editing activity of the CRISPR PLUS proteins.

One of the remaining hypotheses to explore through future research is that chimerically fused GFP might have transformed the Cas9 moiety to conformationally favorable ways, and this resulting C9G protein has increased the turnover rate. Conceptually opposite to the inhibitory activity of anti-CRISPR proteins including AcrIIA2 and AcrIIA $4^{30}$, the fused GFP moiety might interact with Cas9 part so that the C9G proteins better interrogate PAM and target site or release from the target DNA after creation of DSB. Previously, it was shown that, depending on the linker structure, GFP moiety in the chimeric fusion with an acid phosphatase (Pho-C) could differentially affect the enzyme activity ${ }^{31}$. Lastly, understanding the crystal structure of C9 in complex with RecJ or GFP should give us better answer concerning how the chimeric proteins performs better in creation of DNA DSBs.

\section{Conclusion}

In conclusion, we have shown that CRISPR PLUS greatly enhanced genome editing efficiency while keeping the off-target effect low relative to C9 control. Three genes in human HEK293T cells, four in iPSCs, and one in plant cells were tested, and consistently showed that CRISPR PLUS exceeded the editing efficiency of C9. Given that the off-target effects are proportional to duration and concentration of the genome editing enzymes in the cells of treatment ${ }^{11}$, CRISPR PLUS would further decrease the possibility of off-target effects because CRISPR PLUS achieved a genome editing rate within shorter time with relatively lower concentration than those of C9. Greater efficiency is also useful when practicing multiplex genome editing because the possibility to have homozygous alleles simultaneously at the multiple loci equals to the product of the editing rate at an individual locus. Our results should represent breakthrough techniques for multiplex genome editing of the precious and hard-to-edit cells for therapeutics and expedite availability of the regenerative medicines closer. 


\section{Methods}

Cloning of SpyCas9 fusion proteins. The SpyCas9 coding sequence (Addgene, \#138566) was ligated into a pET28a vector (Merck Biosciences, Darmstadt, Germany) that had been linearized by NcoI and XhoI double digestion. The pET28a-SpyCas9 vector, which was named pNGP020, was again linearized by XhoI which was $9 \mathrm{bp}$ downstream of the full-length SpyCas9 sequence. A BPNLS signal peptide and DNA-modifying proteins were introduced to the XhoI site in pNGP020 by digestion of the XhoI site; pNGP020-BPNLS was named pNGP021. Full-length RecJ exonuclease amplified from E. coli DH5a was cloned into the pNGP020 vector by Gibson assembly (NEB, Ipswich, MA), which was then named pNGP022. With the same manner, the GFP gene was amplified from modified GFP4 $4^{32}$, and the SpyCas9-GFP-BPNLS was named pNGP056. RecE exonuclease was amplified from E. coli DH5a, and the SpyCas9-RecE-BPNLS was named pNGP024. T5 phage exonuclease was synthesized by GenScript (Piscataway, NJ, USA), and the SpyCas9-T5-BPNLS was named pNGP041. Lambda phage exonuclease was synthesized by IDT (Coralville, IA, USA), and the SpyCas9-Lambda-BPNLS was named pNGP097. Mung bean exonuclease was amplified from cDNA of mung bean roots purchased from a local grocery store, and the SpyCas9-mungbean exonuclease-BPNLS was named pNGP030. Full-length human TdT (NM_004088) was obtained from the GenScript cDNA library (OHu11174D), and the SpyCas9-hTdTBPNLS was named pNGP072. The DNA sequences mentioned here are provided in Supplementary File 1.

Protein expression and purification. All the vectors encoding SpyCas9 fusion proteins are based on the plasmid vector pET28a backbone and are composed of the SpyCas9, fusion protein, a nucleoplasmin nuclear localization signal (NLS) and 6X-His tag sequences at the N- or C-termini. For bacterial expression of the SpyCas9 chimeric fusion proteins, the plasmids were transformed into Rosetta2 (DE3) pLysS cells. On the first day, E. coli cells harboring the SpyCas9 expression vector from glycerol stocks were inoculated into LB medium containing $50 \mathrm{mg} / \mathrm{L}$ kanamycin and cultured at $37^{\circ} \mathrm{C}$ overnight. On the following day, the growing E. coli cells were reinoculated into fresh $\mathrm{LB}$ medium and incubated at $37^{\circ} \mathrm{C}$ until the optical density at $600 \mathrm{~nm}\left(\mathrm{OD}_{600}\right)$ reached 0.4-0.7, indicating the exponential growth phase. Expression of the SpyCas9 fusion proteins was induced by the addition of isopropyl- $\beta$-D-thiogalactopyranoside (IPTG; final concentration $1 \mathrm{mM}$ ) for $20 \mathrm{~h}$ at $18{ }^{\circ} \mathrm{C}$. Each bacterial culture was centrifuged at $3700 \mathrm{~g}$ for $30 \mathrm{~min}$, and then the pellet was resuspended in lysis buffer $(20 \mathrm{mM}$ Tris- $\mathrm{HCl} \mathrm{pH} \mathrm{8.0,} 500 \mathrm{mM} \mathrm{NaCl}, 5 \mathrm{mM}$ imidazole, $1 \mathrm{mM}$ 1,4-dithiothreitol (DTT), and $1 \mathrm{mM}$ phenylmethylsulfonylfluoride (PMSF)). The resuspended cells were lysed by sonication with a period of $2 \mathrm{~s}$ on and $6 \mathrm{~s}$ off at $40 \%$ amplitude for $40 \mathrm{~min}$ and then centrifuged at $15,000 \mathrm{~g}$ for $1 \mathrm{~h}$. The supernatant was passed through a $0.45 \mu \mathrm{m}$ syringe filter and injected into an AKTA FPLC system (GE Healthcare, Chicago, IL, USA) for purification of the SpyCas9 fusion proteins. Immobilized metal affinity chromatography (IMAC, HisTrap HP 5 mL; GE Healthcare) was performed after washing and equilibrating the AKTA FPLC system. The affinity columns were also washed with ethanol (followed by water) and binding buffer ( $20 \mathrm{mM}$ Tris- $\mathrm{HCl} \mathrm{pH} \mathrm{8.0,500} \mathrm{mM} \mathrm{NaCl,} 5 \mathrm{mM}$ imidazole). The protein was eluted in elution buffer $(20 \mathrm{mM}$ Tris- $\mathrm{HCl} \mathrm{pH} \mathrm{8.0,500} \mathrm{mM} \mathrm{NaCl,} 500 \mathrm{mM}$ imidazole) in a step gradient from 14 to $100 \%$. The flow-through was injected into a desalting column (HiPrep 26/10 Desalting; GE Healthcare) equilibrated with the desalting buffer $(20 \mathrm{mM}$ HEPES, $150 \mathrm{mM} \mathrm{KCl}, 1 \mathrm{mM}$ DTT, $10 \%(\mathrm{v} / \mathrm{v})$ glycerol, $\mathrm{pH} 7.5)$ to replace the imidazole-containing IMAC elution buffer with a final storage buffer to ensure the long-term stability of the eluted proteins. An aliquot of the eluate was subjected to 10\% SDS-PAGE to evaluate protein purity. Finally, the proteins were concentrated in a Vivaspin Turbo 15 concentrator $(50,000$ MWCO; Sartorius, Germany). The concentrated proteins were quantified with the conventional Bradford assay.

In vitro sgRNA transcription. To prepare sgRNAs for in tube cleavage assay, a double-stranded DNA template was generated by annealing two single-stranded oligonucleotides with complementary sequences (forward: 5'-AATTTAATACGACTCACTATAGGXXXXXXXXXXXXXXXXXXXXGTTTTAGAGCTAGAAATAGCA AGTTAAAATAAGGCTAGTCCGTTATCAACTTGAAAAAGTGGCACCGAGTCGGTGCTTTT-3'， reverse: 5'-AAAAGCACCGACTCGGTGCCACTTTTTCAAGTTGATAACGGACTAGCCTTATTTTAACTTGCT ATTTCTAGCTCTAAAACXXXXXXXXXXXXXXXXXXXXCCTATAGTGAGTCGTATTAAATT-3') at $95^{\circ} \mathrm{C}$ for $5 \mathrm{~min}$ and $55^{\circ} \mathrm{C}$ for $10 \mathrm{~min}$. 'X's can be replaced with the spacer sequences for each sgRNA. The DNA was purified using QIAquick (Qiagen) columns, and the purified DNAs were then used as templates for a T7 in vitro transcription (IVT) reaction according to manufacturer's direction (MEGAshortscript T7, Invitrogen, Carlsbad, CA, USA). In vitro transcribed sgRNAs were DNase treated, precipitated using the ammonium acetate/ethanol method, and then resuspended in distilled water for use with the SpyCas9 and its chimeric fusion proteins.

In tube cleavage assay of the SpyCas9 fusion proteins. The CCR5 locus from HEK293T cells was amplified by PCR (Supplementary Table 5) with Q5 High-Fidelity DNA Polymerase (New England Biolabs, Ipswich, MA, USA). For RNP complex formation, 0.25 pmol of each SpyCas9 fusion protein and 0.3 pmol of sgRNA $^{\text {CCR5 }}$ were mixed and incubated at room temperature for $15 \mathrm{~min}$. A total of $250 \mathrm{ng}$ of DNA was digested with preassembled RNP at $37^{\circ} \mathrm{C}$ for $10 \mathrm{~min}$. Digested DNA was placed on ice, and the products were subjected to $2 \%$ agarose gel electrophoresis. Assessment of the digested DNA intensity was performed by Image ${ }^{33}$. The digested fraction was calculated with the following formula: Digested fraction $(\%)=b /(a+b) \times 100$, where $a$ represents the band intensity of the DNA substrate and $b$ represents the digested DNA.

Cell culture and synchronization. HEK293T cells were cultured in Dulbecco's modified Eagle's medium (DMEM; HyClone) supplemented with $10 \%$ fetal bovine serum and cultured at $37^{\circ} \mathrm{C}$ in a humidified $5 \% \mathrm{CO}_{2}$ incubator. HEK293T cells were seeded at $3 \times 10^{6}$ cell density in a $10-\mathrm{cm}$ culture dish. To help improve the efficiency of the knock-in (described below), the cell cycles were synchronized by treatment with nocodazole (200 $\mathrm{ng} / \mathrm{ml})$ for $17 \mathrm{~h}$ before electroporation. 
RNP preparation and nucleofection for knock-in experiments. Before transfection of proteins into the HEK293T cells, purified SpyCas9, C9R, and C9G (30 pmol) and synthetic sgRNA (60 pmol; Thermo Fisher Scientific, Waltham, MA, USA) were incubated at room temperature for 20 min for RNP assembly (sgRNA information, Supplementary Table 4). In the case of the knock-in experiment, 20 pmol ssODN with phosphorothioate bonds in the first and last two nucleotides was then added with the RNP complex. The sequences of the single-stranded oligodeoxynucleotides (ssODNs) used as donor templates for the CCR5, HPRT1, and EMX1 loci are listed in Supplementary Table 3. Each nucleofection reaction consisted of $2 \times 10^{5} \mathrm{HEK} 293 \mathrm{~T}$ cells in $20 \mu \mathrm{l}$ of nucleofection reagent mixed with $10 \mu \mathrm{l}$ of RNP:DNA. For multiplex experiments, 25 pmol of SpyCas9, C9R, or C9G protein and $50 \mathrm{pmol}$ of modified sgRNAs for B2M, CIITA, PD1, or CTLA4 (Supplementary Table 7) (Synthego, Menlo Park, CA, USA) were mixed separately at room temperature for $20 \mathrm{~min}$ before transfection. Nucleofection of HEK293T cells was performed using 4D-Nucleofector (Lonza) with the CM-130 program.

T7 endonuclease I assay. After harvesting samples at $24 \mathrm{~h}$ posttransfection, genomic DNA was extracted using a PureLink Genomic DNA Kit (Thermo Fisher Scientific, Waltham, MA, USA) following the manufacturer's protocol. Primers for the CCR5, HPRT1, and EMX1 loci are listed in Supplementary Table 5. Purified PCR products ( $250 \mathrm{ng}$ ) in NEBuffer 2 (New England Biolabs, Ipswich, MA, USA) were denatured ( $\left(95^{\circ} \mathrm{C}\right.$ for $10 \mathrm{~min}$ ), re-annealed by gradually cooling from 95 to $85^{\circ} \mathrm{C}$ at $-2^{\circ} \mathrm{C} / \mathrm{s}$ and 85 to $25^{\circ} \mathrm{C}$ at $-0.1{ }^{\circ} \mathrm{C} / \mathrm{s}$ and then held at $4{ }^{\circ} \mathrm{C}$ using a programmable thermocycler. The PCR products were treated with $10 \mathrm{U}$ of T7 endonuclease 1 (T7E1; New England Biolabs) in a $20 \mu \mathrm{l}$ final reaction at $37^{\circ} \mathrm{C}$ for $35 \mathrm{~min}$. The product was separated on a $2 \%$ agarose gel. The band intensity was analyzed using ImageJ. The calculation was conducted by the following equation: \% mutation $=100 \times\left(1-(1-f)^{1 / 2}\right)$, where $f$ is the fraction cleaved.

HDR analysis by Ndel restriction digestion. Aa artificially inserted sequence of the NdeI restriction site was PCR amplified (Supplementary Table 5), and the PCR products $(1 \mu \mathrm{g})$ were digested with $20 \mathrm{U}$ of $\mathrm{NdeI}$ (New England Biolabs, Ipswich, MA, USA) at $37^{\circ} \mathrm{C}$ for $3 \mathrm{~h}$. The digested DNA was quenched with $6 \mathrm{X}$ gel loading dye (Purple, New England Biolabs), at $70^{\circ} \mathrm{C}$ for $10 \mathrm{~min}$ and electrophoresed on a $2 \%$ agarose TAE gel. Digested DNA fragment intensity was analyzed and evaluated with ImageJ software.

Targeted deep sequencing of on- and off-target sites. Five potential off-target sites for each gene were predicted using Cas-OFFinder ${ }^{7}$. The genomic region flanking the target site for each gene was amplified by a two-step PCR method. First, genomic DNA from the edited and control samples was isolated and PCR-amplified for 35 cycles using Q5 High-fidelity DNA polymerase with adapter primers. The resulting amplicons were purified using a QIAquick PCR Purification Kit (Qiagen, Venlo, The Netherlands). The samples were subjected to eight cycles of PCR using KAPA HiFi HotStart DNA Polymerase (Roche, Basel, Switzerland) for indexing with MiniSeq High Output Kit (Illumina, FC-420-100X), followed by purification using a QIAquick PCR Purification Kit (Qiagen). Purified DNA samples were quantified with a Qubit 2.0 fluorometer and pooled in an equimolar ratio. Pooled libraries were sequenced in 150 bp read-length with the Illumina MiniSeq (Illumina, SY-420-1001). The PCR primer sequences used to analyze the off-target effects with NGS are listed in Supplementary Table 6.

Validation of C9R exonuclease activity under in vitro conditions. A $60 \mathrm{nM}$ aliquot of either SpyCas9 or C9R was added to the target template, either $125 \mathrm{ng}$ of dsDNA (900 bp) or $3.5 \mu \mathrm{g}$ of ssDNA (123 bp), with or without $\mathrm{Mg}^{2+}$ ions in a $20 \mu \mathrm{l}$ reaction volume. NEBuffer 3.1 (New England Biolabs, Ipswich, MA, USA) was used as the reaction buffer. After $1.5 \mathrm{~h}$ of incubation at $37^{\circ} \mathrm{C}$, the reaction mixtures were assessed by electrophoresis on a $2 \%$ agarose gel.

Plant growth conditions and protoplast transfection of $\boldsymbol{N}$. benthamiana. All plants were grown under $150 \mu \mathrm{mol} \mathrm{m}{ }^{-2} \mathrm{~s}^{-1}$ LED light under long-day (14-h light/10-h dark photoperiod) conditions at $25^{\circ} \mathrm{C}$. Tobacco (Nicotiana benthamiana) seeds were sterilized in a $0.4 \%$ hypochlorite solution for $1 \mathrm{~min}$, washed three times in distilled water, and sown on $0.5 \times$ Gamborg B5 solid medium supplemented with $2 \%$ sucrose. Fourweek-old leaves grown in B5 media were treated with enzymes to digest the cell wall (1.5\% cellulose R10, 0.3\% macerozyme R10, $0.5 \mathrm{M}$ mannitol, $8 \mathrm{mM} \mathrm{CaCl}_{2}, 5 \mathrm{mM}$ MES [pH 5.7], 0.1\% BSA) for $4 \mathrm{~h}$ at $25^{\circ} \mathrm{C}$ in darkness. The mixture was filtered through a $100 \mu \mathrm{m}$ cell strainer (Thermo Fisher Scientific, Waltham, MA, USA), and then flow-through protoplasts were collected by centrifugation at $100 \mathrm{~g}$ in a round-bottom tube for $6 \mathrm{~min}$. Protoplasts were resuspended and washed with W5 solution $\left(154 \mathrm{mM} \mathrm{NaCl}, 125 \mathrm{mM} \mathrm{CaCl} \mathrm{Cl}_{2} \cdot 2 \mathrm{H}_{2} \mathrm{O}, 5 \mathrm{mM} \mathrm{KCl}, 2 \mathrm{mM}\right.$ MES pH 5.7) and pelleted by centrifugation at $100 \mathrm{~g}$ for $6 \mathrm{~min}$. Finally, protoplasts were resuspended in MMG solution (0.4 M mannitol, $15 \mathrm{mM} \mathrm{MgCl}, 4 \mathrm{mM} \mathrm{MES} \mathrm{pH} \mathrm{5.7)} \mathrm{and} \mathrm{counted} \mathrm{under} \mathrm{a} \mathrm{microscope} \mathrm{using} \mathrm{a} \mathrm{hemo-}$ cytometer. Protoplasts were diluted to a density of $1 \times 10^{6}$ protoplasts $/ \mathrm{ml}$ of MMG solution and stabilized for at least $30 \mathrm{~min}$ at $4{ }^{\circ} \mathrm{C}$ before PEG-mediated transfection.

Protoplasts $\left(2 \times 10^{5}\right)$ were transfected with SpyCas9 and C9R proteins $(10 \mu \mathrm{g})$ premixed with in vitro-transcribed sgRNA $(20 \mu \mathrm{g})$ targeting $\alpha$-1,3-fucosyltransferase 1 (FucT 13-1) (Supplementary Table 4). Prior to transfection, SpyCas9 protein was mixed with sgRNA in $1 \times$ NEB buffer 3 and incubated for $10 \mathrm{~min}$ at room temperature. A mixture of protoplasts was resuspended in $200 \mu \mathrm{MMG}$ solution and gently mixed with $10-20 \mu \mathrm{l}$ of RNP complex and 210-220 $\mu \mathrm{l}$ of freshly prepared PEG (0.2 M mannitol, 40\% w/v PEG-4000, $100 \mathrm{mM} \mathrm{CaCl}_{2}$ ) solution and then incubated at $25^{\circ} \mathrm{C}$ for $15 \mathrm{~min}$. After a $15 \mathrm{~min}$ incubation at room temperature, transformation was stopped by adding $840-880 \mu \mathrm{l}$ of W5 solution. Protoplasts were then collected by centrifugation for $2 \mathrm{~min}$ at $100 \mathrm{~g}$ at room temperature, washed once with $1 \mathrm{ml}$ of wash buffer, and then collected by centrifuging for another $2 \mathrm{~min}$ at $100 \mathrm{~g}$. The density was adjusted to $1 \times 10^{5}$ protoplasts $/ \mathrm{ml}$, and they were cultured in modified Protoplast 
Induction Medium (PIM) medium (1.58 g B5 medium, 103 g sucrose, $0.2 \mathrm{mg}$ 2,4-D, $0.3 \mathrm{mg}$ 6-Benzylaminopurine (BAP), $0.1 \mathrm{~g} \mathrm{MES}, 375 \mathrm{mg} \mathrm{CaCl} 2 \cdot 2 \mathrm{H}_{2} \mathrm{O}, 18.35 \mathrm{mg} \mathrm{NaFe}$-EDTA and $270 \mathrm{mg}$ sodium succinate).

iPSC reprogramming and maintenance. Human dermal fibroblasts (DFs; ATCC, Manassas, VA, US) were plated in 24-well plates. After one day, the cells were transduced with reprogramming factors (Oct4, Sox2, $\mathrm{Klf}-4$, and c-Myc) in $5 \mu \mathrm{g} / \mathrm{mL}$ polybrene (Sigma, St. Louis, MO, US) based on a multiplicity of infection 10 . After 5 days, transduced cells were transferred to STO feeder cells (ATCC) in feeder medium [DMEM/F12 (Thermo Fisher Scientific, Waltham, MA, US) supplemented with 10\% FBS (Thermo) and $1 \times$ primocin (InvivoGen, San Diego, CA, US)]. After 2 days, medium exchange was initiated with iPSC medium [DMEM/F12 supplemented with $20 \%$ serum replacement (Thermo), $1 \times$ primocin and $4 \mathrm{ng} / \mathrm{mL}$ bFGF (Thermo)]. Newly-formed colonies were consistently subcultured by mechanical picking under a stereoscopic microscope. Picked colonies were maintained on STO feeder cells and mechanically passaged to fresh feeder cells every 7 days. DF-derived iPSCs were used for further gene knockout (KO) experiments.

Multiplex genome editing in iPSCs. For gene KO, RNP mixtures of CRISPR sgRNA (B2M, CIITA, $P D C D-1$, and CTLA-4; each $50 \mathrm{pmol}$ ) and nuclease protein (C9, C9R, and C9G; each 25 pmol) were introduced into iPSCs using the Neon transfection system (Thermo, Waltham, MA, US). sgRNA information for multiplex gene knockout is listed in Supplementary Table 7. After the introduction of the RNP mixture, iPSCs were cultured for $1 \mathrm{~d}$ or $3 \mathrm{~d}$ in mTeSR Plus medium (Stemcell Technologies, Vancouver, BC, CA) on Matrigel (Corning, New York, MA, US)-coated culture plates. Cells were pooled after $1 \mathrm{~d}$ or $3 \mathrm{~d}$ of electroporation and used for subsequent analysis.

Statistical analyses. Results were analyzed using GraphPad (ver. Prime 9). Comparison of groups was analyzed using a Nested t-test and ANOVA.

Received: 22 March 2021; Accepted: 19 July 2021

Published online: 10 August 2021

\section{References}

1. Jinek, M. et al. A programmable dual-RNA-guided DNA endonuclease in adaptive bacterial immunity. Science 337, 816-821. https://doi.org/10.1126/science.1225829 (2012).

2. Makarova, K. S. et al. Evolutionary classification of CRISPR-Cas systems: A burst of class 2 and derived variants. Nat. Rev. Microbiol. 18, 67-83. https://doi.org/10.1038/s41579-019-0299-x (2020).

3. Pausch, P. et al. CRISPR-CasPhi from huge phages is a hypercompact genome editor. Science 369, 333-337. https://doi.org/10. 1126/science.abb1400 (2020).

4. Huang, C. H., Lee, K. C. \& Doudna, J. A. Applications of CRISPR-Cas enzymes in cancer therapeutics and detection. Trends Cancer 4, 499-512. https://doi.org/10.1016/j.trecan.2018.05.006 (2018).

5. Zhu, H., Li, C. \& Gao, C. Applications of CRISPR-Cas in agriculture and plant biotechnology. Nat. Rev. Mol. Cell. Biol. 21, 661-677. https://doi.org/10.1038/s41580-020-00288-9 (2020).

6. Pickar-Oliver, A. \& Gersbach, C. A. The next generation of CRISPR-Cas technologies and applications. Nat. Rev. Mol. Cell. Biol. 20, 490-507. https://doi.org/10.1038/s41580-019-0131-5 (2019).

7. Bae, S., Park, J. \& Kim, J. S. Cas-OFFinder: A fast and versatile algorithm that searches for potential off-target sites of Cas9 RNAguided endonucleases. Bioinformatics 30, 1473-1475. https://doi.org/10.1093/bioinformatics/btu048 (2014).

8. Donohoue, P. D., Barrangou, R. \& May, A. P. Advances in industrial biotechnology using CRISPR-Cas systems. Trends Biotechnol. 36, 134-146. https://doi.org/10.1016/j.tibtech.2017.07.007 (2018).

9. Scully, R., Panday, A., Elango, R. \& Willis, N. A. DNA double-strand break repair-pathway choice in somatic mammalian cells. Nat. Rev. Mol. Cell. Biol. 20, 698-714. https://doi.org/10.1038/s41580-019-0152-0 (2019).

10. Sander, J. D. \& Joung, J. K. CRISPR-Cas systems for editing, regulating and targeting genomes. Nat. Biotechnol. 32, 347-355. https:// doi.org/10.1038/nbt.2842 (2014).

11. Kim, D., Luk, K., Wolfe, S. A. \& Kim, J. S. Evaluating and enhancing target specificity of gene-editing nucleases and deaminases. Annu. Rev. Biochem. 88, 191-220. https://doi.org/10.1146/annurev-biochem-013118-111730 (2019).

12. Filippova, J., Matveeva, A., Zhuravlev, E. \& Stepanov, G. Guide RNA modification as a way to improve CRISPR/Cas9-based genome-editing systems. Biochimie 167, 49-60. https://doi.org/10.1016/j.biochi.2019.09.003 (2019).

13. Sternberg, S. H., Redding, S., Jinek, M., Greene, E. C. \& Doudna, J. A. DNA interrogation by the CRISPR RNA-guided endonuclease Cas9. Nature 507, 62-67. https://doi.org/10.1038/nature13011 (2014).

14. Clements, T. P., Tandon, B., Lintel, H. A., McCarty, J. H. \& Wagner, D. S. RICE CRISPR: Rapidly increased cut ends by an exonuclease Cas9 fusion in zebrafish. Genesis https://doi.org/10.1002/dvg.23044 (2017).

15. Certo, M. T. et al. Coupling endonucleases with DNA end-processing enzymes to drive gene disruption. Nat. Methods 9, 973-975. https://doi.org/10.1038/nmeth.2177 (2012).

16. Cermak, T. et al. A multipurpose toolkit to enable advanced genome engineering in plants. Plant Cell 29, 1196-1217. https://doi. org/10.1105/tpc.16.00922 (2017).

17. Wu, Y. et al. Improving FnCas12a genome editing by exonuclease fusion. CRISPR J. 3, 503-511. https://doi.org/10.1089/crispr. 2020.0073 (2020).

18. Charpentier, M. et al. CtIP fusion to Cas9 enhances transgene integration by homology-dependent repair. Nat. Commun. 9, 1133 . https://doi.org/10.1038/s41467-018-03475-7 (2018).

19. Jayavaradhan, R. et al. CRISPR-Cas9 fusion to dominant-negative 53BP1 enhances HDR and inhibits NHEJ specifically at Cas9 target sites. Nat. Commun. 10, 2866. https://doi.org/10.1038/s41467-019-10735-7 (2019).

20. Shao, S. et al. Enhancing CRISPR/Cas9-mediated homology-directed repair in mammalian cells by expressing Saccharomyces cerevisiae Rad52. Int. J. Biochem. Cell Biol. 92, 43-52. https://doi.org/10.1016/j.biocel.2017.09.012 (2017).

21. Kim, Y. B. et al. Increasing the genome-targeting scope and precision of base editing with engineered Cas9-cytidine deaminase fusions. Nat. Biotechnol. 35, 371-376. https://doi.org/10.1038/nbt.3803 (2017). 
22. Gaudelli, N. M. et al. Publisher correction: Programmable base editing of $A^{*} T$ to $G^{*} C$ in genomic DNA without DNA cleavage. Nature 559, E8. https://doi.org/10.1038/s41586-018-0070-x (2018).

23. Anzalone, A. V. et al. Search-and-replace genome editing without double-strand breaks or donor DNA. Nature 576, 149-157. https://doi.org/10.1038/s41586-019-1711-4 (2019).

24. Kowalczykowski, S. C., Dixon, D. A., Eggleston, A. K., Lauder, S. D. \& Rehrauer, W. M. Biochemistry of homologous recombination in Escherichia coli. Microbiol. Rev. 58, 401-465 (1994).

25. Vakulskas, C. A. et al. A high-fidelity Cas9 mutant delivered as a ribonucleoprotein complex enables efficient gene editing in human hematopoietic stem and progenitor cells. Nat. Med. 24, 1216. https://doi.org/10.1038/s41591-018-0137-0 (2018).

26. Lin, S., Staahl, B. T., Alla, R. K. \& Doudna, J. A. Enhanced homology-directed human genome engineering by controlled timing of CRISPR/Cas9 delivery. Elife 3, e04766. https://doi.org/10.7554/eLife.04766 (2014).

27. Koga, K., Wang, B. \& Kaneko, S. Current status and future perspectives of HLA-edited induced pluripotent stem cells. Inflamm. Regen. 40, 23. https://doi.org/10.1186/s41232-020-00132-9 (2020).

28. Ren, J. et al. A versatile system for rapid multiplex genome-edited CAR T cell generation. Oncotarget 8, 17002-17011. https://doi. org/10.18632/oncotarget.15218 (2017).

29. Woo, J. W. et al. DNA-free genome editing in plants with preassembled CRISPR-Cas9 ribonucleoproteins. Nat. Biotechnol. 33, 1162-1164. https://doi.org/10.1038/nbt.3389 (2015).

30. Dong, et al. Structural basis of CRISPR-SpyCas9 inhibition by an anti-CRISPR protein. Nature 546, 436-439. https://doi.org/10. 1038/nature22377 (2017).

31. Huang, Z., Li, G., Zhang, C. \& Xing, X. H. A study on the effects of linker flexibility on acid phosphatase PhoC-GFP fusion protein using a novel linker library. Enzyme Microb. Technol. 83, 1-6. https://doi.org/10.1016/j.enzmictec.2015.11.002 (2016).

32. Haseloff, J., Siemering, K. R., Prasher, D. C. \& Hodge, S. Removal of a cryptic intron and subcellular localization of green fluorescent protein are required to mark transgenic Arabidopsis plants brightly. Proc. Natl. Acad. Sci. U. S. A. 94, 2122-2127. https://doi.org/ 10.1073/pnas.94.6.2122 (1997).

33. Rueden, C. T. et al. Image J2: ImageJ for the next generation of scientific image data. BMC Bioinformatics 18, 529. https://doi.org/ 10.1186/s12859-017-1934-z (2017)

\section{Acknowledgements}

This project has been supported by the CRISPR PLUS R\&D program (2016GFRND-CP001) supported by G+FLAS Life Sciences, Inc., and Basic Science Research Program through the National Research Foundation of Korea (NRF) funded by the Ministry of Science and ICT (2020R1F1A1063608).

\section{Author contributions}

S.C. And K.-S.K. supervised the research. J.P. designed and created the CRISPR PLUS constructs. J.Y. and S.P. purified proteins. S.Choi performed plant protoplast experiments. Ja.L. and Ju.L. performed efficiency and NGS analysis. K.L. designed the guide RNA and performed bioinformatics analysis. D.K., M-J.H., S.L. designed and performed the iPSCs study. K-S.K. supervised the iPSCs study and contributed to writing. S.C. designed the constructs and wrote the paper.

\section{Competing interests}

J.P. is an employee of Naturegenic, Inc., and J.Y., S.Choi., Ju.L., S.P., K.L., and Ja.L. are employees of G + FLAS Life Sciences. S.C., J.P., J.Y., Ju.L., and Ja.L. are inventors of patents and patent applications related to the CRISPR PLUS genome editing technologies. S.Choe. is a CEO of G + FLAS Life Sciences, Inc. K-S.K. is a founder and GVO (Global Vision Officer) of Kangstem Biotech Co., Ltd., D.K., M-J.H., S.L. is employees of Kangstem Biotech Co., Ltd. All other authors declare no conflicts of interests.

\section{Additional information}

Supplementary Information The online version contains supplementary material available at https://doi.org/ 10.1038/s41598-021-95406-8.

Correspondence and requests for materials should be addressed to K.-S.K. or S.C.

Reprints and permissions information is available at www.nature.com/reprints.

Publisher's note Springer Nature remains neutral with regard to jurisdictional claims in published maps and institutional affiliations.

Open Access This article is licensed under a Creative Commons Attribution 4.0 International License, which permits use, sharing, adaptation, distribution and reproduction in any medium or format, as long as you give appropriate credit to the original author(s) and the source, provide a link to the Creative Commons licence, and indicate if changes were made. The images or other third party material in this article are included in the article's Creative Commons licence, unless indicated otherwise in a credit line to the material. If material is not included in the article's Creative Commons licence and your intended use is not permitted by statutory regulation or exceeds the permitted use, you will need to obtain permission directly from the copyright holder. To view a copy of this licence, visit http://creativecommons.org/licenses/by/4.0/.

(C) The Author(s) 2021, corrected publication 2021 